Journal of Computer Science 7 (2): 197-205, 2011

ISSN 1549-3636

(C) 2011 Science Publications

\title{
Synchronization of Chaos Systems Using Fuzzy Logic
}

\author{
${ }^{1}$ Mohammad Ababneh, ${ }^{2}$ Issa Etier, \\ ${ }^{2}$ Mahmoud Smadi and ${ }^{2}$ Jasim Ghaeb \\ ${ }^{1}$ Department of Mechatronics Engineering, \\ ${ }^{2}$ Department of Electrical Engineering, \\ College of Engineering, the Hashemite University, 13115 Zarqa, Jordan
}

\begin{abstract}
Problem statement: This study presented a new and systematic method to robustly synchronize uncertain chaos systems. It was derived based on the observer approach for synchronization, where error dynamics was made asymptotically stable around the zero to accomplish synchronization. Approach: This method viewed the synchronization problem as a control problem in order to make use of the literature available in this field. In addition, it consisted of designing a digital response system to synchronize with a given continuous-time chaotic drive system. Takagi-Sugeno (TS) fuzzy model was used to model the chaotic dynamic system, while the uncertainties were decomposed such that the uncertain chaotic system could be rewritten as a set of local linear models with an additional disturbed input. Results: This study demonstrated the effectiveness of the methodology presented. The response (receiver) system was able to synchronize very closely with drive (transmitter) system. Furthermore, both piecewise linear and nonlinear uncertain Chua circuits synchronized wonderfully with insignificant errors. Conclusion: The study confirmed that it is capable of achieving excellent synchronization performances, even in the presence of significant parametric uncertainties for uncertain chaos systems. The Takagi-Sugeno (TS) fuzzy model was used to model the chaotic dynamic system, while the uncertainties are decomposed such that the uncertain chaotic system was rewritten as a set of local linear models with an additional disturbed input.
\end{abstract}

Key words: Takagi-Sugeno (TS), using fuzzy logic, drive system, dynamic system, proposed method, synchronization problem, linear model, chaotic system, uncertain parameters

\section{INTRODUCTION}

Real world systems are uncertain in general. However, it is a common practice to describe a real system using nominal linear models; the primary reason to do this is the advantage of using the well-developed linear techniques for analysis and design of the controller. Unfortunately, under some conditions like uncertainties, modeling errors, noise and other disturbances, the nominal linear approach might fail to produce satisfactory results. To overcome this problem, it is critical to design and implement systems that take into consideration these aspects of the system's dynamics. A more suitable representation of a real world system is a continuous-time parametric-uncertain model with bounded disturbances and noise inputs.

Generally, synchronization is when two different systems come to behave in accordance as a function of time, this occurs as the result of some kind of interaction between them. Recently, chaos synchronization has been an active research area (Yau and Yan, 2008; Bowong et al., 2006; Xie et al., 2002). Where chaos synchronization of different chaotic systems, such as Lorenz-Chen, Chen-Liu and LiuLorenz, in drive-response structure were considered. Therefore, many chaos synchronization methodologies have been proposed in the literature. One important approach is to consider is the synchronization problem from the point of view of control theory (Blekhman et al., 2002; Nijmeijer, 2001; Nijmeijer et al., 1997). Where synchronization was achieved with a drive chaotic system through coupling it with response .

For this representation, most of the research efforts have been concentrated on the design of continuoustime controllers for continuous-time systems or digital controllers for digital systems. In this study we consider the hybrid case; our objective is to design a digital controller for a continuous-time uncertain chaotic system with structured uncertainties using fuzzy logic.

The idea of developing a digital controller from a previously designed continuous-time one has been utilized in (Ababneh et al., 2007), where a controller is

Corresponding Author: Mohammad Ababneh, College of Engineering, the Hashemite University, 13115 Zarqa, Jordan 
designed in terms of the optimal linear model representation of the nominal system around each operating point of the trajectory. Also, digital controllers had been applied to different types of systems, among them are PWM controllers as in (Fujioka et al., 2009), cascaded analog controllers as in (Shieh et al., 1998), where digital redesign method is used to find new pulse-amplitude-modulated (PAM) and pulse-width-modulated (PWM) digital controllers for effective digital control of the analog system, Also delayed systems. In addition, a new intelligent digital redesign method for uncertain nonlinear systems is presented in (Sung et al., 2010) and designing digital controllers for uncertain chaotic systems using fuzzy logic was presented in (Ababneh et al., 2009).

The application of digital redesign to chaotic systems was proposed on earlier works (Ababneh et al., 2007), where designing different digital controllers for uncertain chaotic systems was implemented. However, the problem of applying digital redesign technique to synchronize chaotic systems with uncertainties using fuzzy modeling has not been addressed. On this regard, the methodology proposed in this study is to decompose and incorporate the uncertainty in the system. Next, using Takagi-Sugeno (TS) fuzzy modeling (Bernal et al., 2009; Ahmad and Mohamed, 2009; Hafaifa et al., 2009; Hassan et al., 2010), the uncertain chaotic system is expressed as set of linear models, then, for each linear model synchronization performed as the solution to a linear matrix inequality problem.

Fuzzy modeling: Here we will discuss the fuzzy modeling of chaotic systems and more specifically the Takagi-Sugeno (TS) fuzzy model is used. In addition, Chua's circuit is utilized to demonstrate the application of TS model to chaotic system modeling. We represent the chaotic system dynamics by set of local relations in the state space, The TS model represents every fuzzy rule by a linear model.

Consider a family of chaotic systems of the form:

$\dot{x}(t)=f(x)+g(x) u(t)$

where, $\quad f: \mathfrak{R}^{\mathrm{n}} \rightarrow \mathfrak{R}^{\mathrm{n}}$ and $\mathrm{g}: \mathfrak{R}^{\mathrm{n}} \rightarrow \mathfrak{R}^{\mathrm{n} \times \mathrm{m}}$ are chaotic functions, $x(t) \in \Re^{n}$ is the state vector and $u(t) \in \Re^{m}$ is the control input.

The TS system is described by IF-THEN statement (Bernal et al., 2009). Every rule represents a linear model of the system as follows:

Rule $\mathrm{j}: \operatorname{IFx}_{1}(\mathrm{t})$ is $\mathrm{M}_{\mathrm{I}}^{\mathrm{j}}$... and $\mathrm{x}_{\mathrm{n}}(\mathrm{t})$ is $\mathrm{M}_{\mathrm{n}}^{\mathrm{j}}$

Then $\dot{x}(t)=A_{j} x(t)+B_{j} u(t)$ where, $j=1,2, \ldots, q, x(t)=\left[x_{1}, x_{2}, \ldots x_{n}\right]^{\gamma}, u\left[u_{1}, u_{2}, u_{m}\right]^{\gamma}$. The $\mathrm{M}_{\mathrm{j}}$ is the fuzzy set and $\mathrm{q}$ is the number of rules. Given a pair of $(x(t), u(t))$, the fuzzy system is inferred as following:

$\dot{x}(t)=A_{j} x(t)+B_{j} u(t)$

where, $A=\sum_{j-1}^{q} \mu(x) A_{j}, B=\sum_{j-1}^{q} \mu(x) B_{j}$ and:

$\mu_{j}=\frac{\omega_{j}(x)}{\sum_{j-1}^{q} \omega_{j}(x)} \geq 0, \sum_{j-1}^{q} \omega_{j}(x)=1, \omega_{j}(x)=\prod_{j-1}^{q} M_{j}^{1}$

The input system is given by:

$\dot{x}(t)=\frac{\sum_{j-1}^{q} \omega_{j}(x)\left(A_{j} x(t)+B_{j} u(y)\right)}{\sum_{j-1}^{q} \omega_{j}(t)}$

Fuzzy modeling of Chua's chaotic circuits are developed as explained below. The electronic circuit of Fig. 1 is the realization of one of the most widely used benchmarks for chaotic dynamics (Gámez-Guzmán et al., 2009).

There are different mathematical representations of Chua's circuit, in this study we will adopt the representation in (Lu et al., 2004) as follows:

$$
\begin{aligned}
& \dot{\mathrm{v}}_{1}(\tau)=\frac{1}{\mathrm{C}_{1}}\left[\frac{1}{\mathrm{R}}\left(\mathrm{v}_{2}(\tau)-\mathrm{v}_{1}(\tau)\right)-\mathrm{f}_{\mathrm{NL}}\left(\mathrm{v}_{1}(\tau)\right)\right] \\
& \dot{\mathrm{v}}_{2}(\tau)=\frac{1}{\mathrm{C}_{2}}\left[\frac{1}{\mathrm{R}}\left(\mathrm{v}_{1}(\tau)-\mathrm{v}_{2}(\tau)+\mathrm{i}_{1}(\tau)\right)\right] \dot{\mathrm{i}}_{1}(\tau)=\frac{1}{\mathrm{~L}} \mathrm{v}_{2}(\tau)
\end{aligned}
$$

Chua's circuit has two forms, these are the piecewise linear and the nonlinear forms, which are considered below.

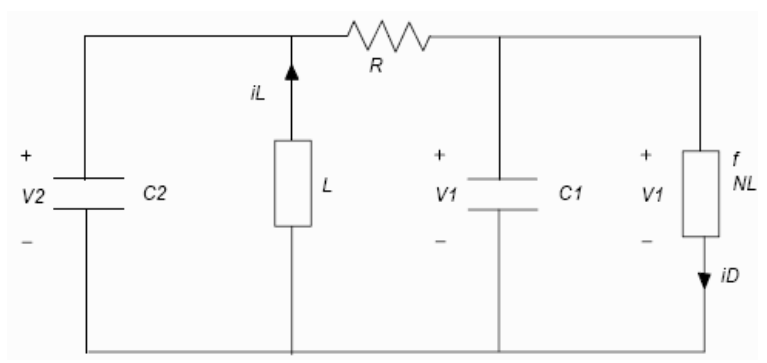

Fig. 1: Chua's circuit realization 
Piecewise-linear case: In the piecewise-linear case, $\mathrm{f}_{\mathrm{NL}}$ represents the nonlinear resistance of the circuit, which is represented by a piecewise-linear function and expressed as follows:

$$
\begin{aligned}
& \mathrm{f}_{\mathrm{NL}}\left(\mathrm{v}_{1}(\tau)\right)=\mathrm{g}_{\mathrm{b}} \mathrm{v}_{1}(\tau)+\frac{1}{2}\left(\mathrm{~g}_{\mathrm{a}}-\mathrm{g}_{\mathrm{b}}\right) \\
& \left(\left|\mathrm{v}_{1}(\tau)+\mathrm{E}\right|-\left|\mathrm{v}_{1}(\tau)-\mathrm{E}\right|\right)
\end{aligned}
$$

where, $g_{a} g_{b}<0$, Or it can be expressed more conveniently as:

$$
f_{N L}\left(v_{1}(\tau)\right)=\left\{\begin{array}{cc}
g_{b} v_{1}(\tau)+\left(g_{a}-g_{b}\right) E & v_{1}(\tau) \geq E \\
g_{a} v_{1}(\tau) & -E<v_{1}(\tau)<E \\
g_{b} v_{1}(\tau)-\left(g_{a}-g_{b}\right) E & v_{1}(\tau) \leq-E
\end{array}\right\}
$$

At this point we need to obtain the fuzzy model of Chua's chaotic system. Considering $\mathrm{f}_{\mathrm{NL}}\left(\mathrm{v}_{1}(\tau)\right) \in[-\mathrm{d}, \mathrm{d}], \mathrm{d}>\mathrm{E}>0$ as shown in Fig. 2, we obtain the following bounds:

$$
\begin{aligned}
& \mathrm{f}_{\mathrm{a}}\left(\mathrm{v}_{1}(\tau)\right)=\mathrm{g}_{\mathrm{a}} \mathrm{v}_{1}(\tau) \\
& \mathrm{f}_{\mathrm{b}}\left(\mathrm{v}_{1}(\tau)\right)=\left(\mathrm{g}_{\mathrm{a}} \mathrm{v}_{1}+\frac{\left(\mathrm{g}_{\mathrm{a}}-\mathrm{g}_{\mathrm{b}}\right) \mathrm{E}}{\mathrm{d}}\right)\left(\mathrm{v}_{1}(\tau)\right)=\mathrm{G}_{\mathrm{ab}}\left(\mathrm{v}_{1}(\tau)\right)
\end{aligned}
$$

When $g_{a}=g_{b}$, the Chua's system becomes linear, otherwise we use the trapezoidal membership functions shown in Fig. 3 to model the Chua's system.

Assigning state vector as $\mathrm{x}=\left[\mathrm{vc}_{1}, \mathrm{vc}_{2}, \mathrm{i}_{1}\right]$, Chua's circuit of (5) can be represented using the following model:

Rule 1: $\operatorname{IFv}_{1}(t)$ is $\mathrm{M}_{1}\left(\mathrm{v}_{1}\right)($ near 0$)$

Then $\dot{\mathrm{x}}(\mathrm{t})=\mathrm{A}_{1} \mathrm{x}(\mathrm{t})+\mathrm{B}_{1} \mathrm{u}(\mathrm{t})$

Rule 2: $\operatorname{IFv}_{1}(\mathrm{t})$ is $\mathrm{M}_{2}\left(\mathrm{v}_{1}\right)($ near $\pm \mathrm{d})$

Then $\dot{x}(t)=A_{2} x(t)+B_{2} u(t)$

Where:

$$
\begin{aligned}
A_{1} & =\left(\begin{array}{ccc}
-\frac{1}{C_{1} R}-\frac{g_{a}}{C_{1}} & \frac{1}{C_{1} R} & 0 \\
\frac{1}{C_{1} R} & -\frac{1}{C_{1} R} & \frac{1}{C_{1}} \\
0 & -\frac{1}{L} & 0
\end{array}\right), \\
A_{2} & =\left(\begin{array}{ccc}
-\frac{1}{C_{1} R}-\frac{g_{a b}}{C_{1}} & \frac{1}{C_{1} R} & 0 \\
\frac{1}{C_{2} R} & -\frac{1}{C_{2} R} & \frac{1}{C_{2}} \\
0 & -\frac{1}{L} & 0
\end{array}\right) m_{2}=m_{c} R
\end{aligned}
$$

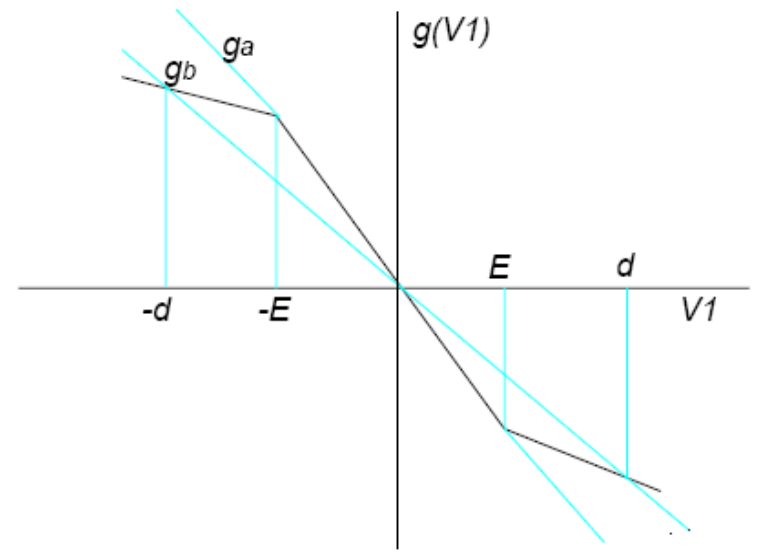

Fig. 2: Piecewise-linear representation of the Chua's resistor circuit realization

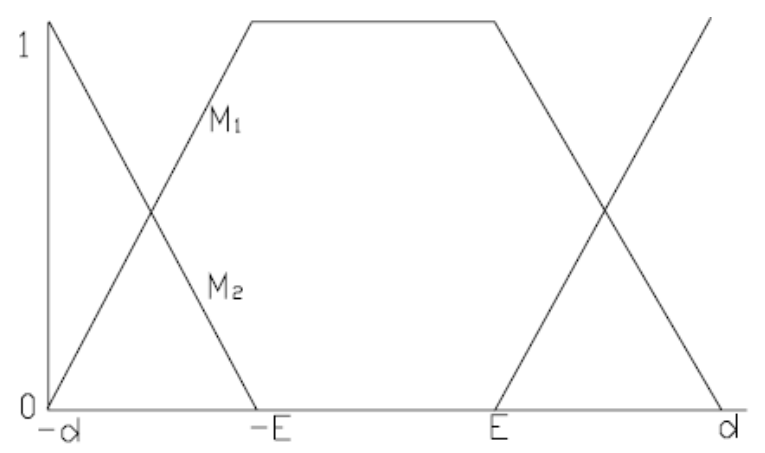

Fig. 3: Membership functions

and $\mathrm{B}_{1}=\mathrm{B}_{2}=\mathrm{I}_{3 \times 3} \quad$ substituting $\mathrm{a}=\frac{1}{\mathrm{C}_{1} \mathrm{R}}$ and $\mathrm{b}=\frac{1}{\mathrm{~L}} \quad$ will result in:

$$
\begin{aligned}
& \mathrm{A}_{1}=\left(\begin{array}{ccc}
-\mathrm{a}\left(1-\frac{\mathrm{g}_{\mathrm{a}}}{\mathrm{C}_{1}}\right) & \mathrm{a} & 0 \\
1 & -1 & 1 \\
0 & -\mathrm{b} & 0
\end{array}\right), \\
& \mathrm{A}_{2}=\left(\begin{array}{ccc}
-\mathrm{a}\left(1-\frac{\mathrm{g}_{\mathrm{ab}}}{\mathrm{C}_{1}}\right) & \mathrm{a} & 0 \\
1 & -1 & 1 \\
0 & -\mathrm{b} & 0
\end{array}\right)
\end{aligned}
$$

Nonlinear case: In the nonlinear case NL f represents the nonlinear resistance as follows $\mathrm{g}_{\mathrm{NL}}\left(\mathrm{x}_{1}(\mathrm{t})\right)=\mathrm{m}_{1} \mathrm{x}_{1}(\mathrm{t})+\mathrm{m}_{2} \mathrm{x}_{1}(\mathrm{t})^{3}$ for $\mathrm{m}_{1}=\mathrm{m}_{\mathrm{a}} \mathrm{R}$ and $\mathrm{m}_{2}=\mathrm{m}_{\mathrm{c}} \mathrm{R}$. 
As in the previous case, consider $\mathrm{f}_{\mathrm{NL}}\left(\mathrm{v}_{1}(\tau)\right) \in[-\mathrm{d}, \mathrm{d}], \mathrm{d}>\mathrm{E}$, we obtain the following bounds

$\mathrm{g}_{1}\left(\mathrm{v}_{1}(\tau)\right)=\mathrm{m}_{\mathrm{a}} \mathrm{v}_{1}(\tau), \mathrm{g}_{2}\left(\mathrm{v}_{1}(\tau)\right)=\left(\mathrm{m}_{1}+\mathrm{m}_{2} \mathrm{~d}^{2}\right) \mathrm{v}_{1}(\tau)=\mathrm{m}_{12} \mathrm{v}_{1}(\tau)$.

The membership function is given by (Bernal et al., 2009) can be written as follows:

$$
\begin{aligned}
& M_{1}\left(v_{1}(\tau)\right)=1-\left(\frac{v_{1}(\tau)}{d}\right)^{2}, M_{2}\left(v_{1}(\tau)\right) \\
& =1-M_{1}\left(v_{1}(\tau)\right)=\left(\frac{v_{1}(\tau)}{d}\right)^{2}
\end{aligned}
$$

Then the Chua's circuit can be represented with:

Rule $1: \operatorname{IFv}_{1}(\mathrm{t})$ is $\mathrm{M}_{1}\left(\mathrm{v}_{1}\right)($ near 0$)$

Then $\dot{\mathrm{x}}(\mathrm{t})=\mathrm{A}_{1} \mathrm{x}(\mathrm{t})+\mathrm{B}_{1} \mathrm{u}(\mathrm{t})$

Rule 2: $\operatorname{IFv}_{1}(\mathrm{t})$ is $\mathrm{M}_{2}\left(\mathrm{v}_{1}\right)($ near $\pm \mathrm{d})$

Then $\dot{x}(t)=A_{2} x(t)+B_{2} u(t)$

Where:

$$
\begin{aligned}
A_{1} & =\left(\begin{array}{ccc}
-\frac{1}{C_{1} R}-\frac{m_{a}}{C_{1}} & \frac{1}{C_{1} R} & 0 \\
\frac{1}{C_{2} R} & -\frac{1}{C_{2} R} & \frac{1}{C_{2}} \\
0 & -\frac{1}{L} & 0
\end{array}\right), \\
A_{2} & =\left(\begin{array}{ccc}
-\frac{1}{C_{1} R}-\frac{m_{12}}{C_{1}} & \frac{1}{C_{1} R} & 0 \\
\frac{1}{C_{2} R} & -\frac{1}{C_{2} R} & \frac{1}{C_{2}} \\
0 & -\frac{1}{L} & 0
\end{array}\right),
\end{aligned}
$$

and $B_{1}-B_{2}=I_{3 \times 3} \quad$ substituting $a=\frac{1}{C_{1} R}$ and $b=\frac{1}{L} \quad$ will lead to:

$$
A_{1}=\left(\begin{array}{ccc}
-a-\frac{m_{a}}{C_{1}} & a & 0 \\
1 & -1 & 1 \\
0 & -b & 0
\end{array}\right), A_{2}=\left(\begin{array}{ccc}
-a-\frac{m_{12}}{C_{1}} & a & 0 \\
1 & -1 & 1 \\
0 & -b & 0
\end{array}\right)
$$

Uncertainty decomposition: In what follows, we present a method to incorporate the uncertainties of the chaotic system in the fuzzy linear model. In particular, we consider the case of parametric variations. This type of disturbance can be seen as a structured uncertainty of the system. This method consist of representing the uncertain chaotic system as a set of uncertain local linear models, then the uncertainty is decomposed and incorporated into the local models such that they can be rewritten as nominal linear models with an additional disturbance input.

Considering the following uncertain chaotic system of Eq. 1 and applying the fuzzy modeling discussed earlier the uncertain chaotic model of Eq. 9 can be represented by a set of local uncertain linear models of the form:

$$
\begin{aligned}
& x_{c}(t)=\tilde{A}_{j} x_{c}(t)+\tilde{B}_{j} u_{c}(t)=\left(A_{a, j} \pm \Delta A_{j}\right) x_{c}(t) \\
& +\left(B_{a, j} \pm \Delta B_{j}\right) u_{c}(t)
\end{aligned}
$$

where, $\quad \mathrm{A}_{0, \mathrm{j}} \in \mathfrak{R}_{\mathrm{n} \times \mathrm{n}}$ and $\mathrm{B}_{0, \mathrm{j}} \in \mathfrak{R}_{\mathrm{n} \times \mathrm{m}}$ are the nominal system and nominal input matrices respectively, while $\Delta \mathrm{A}_{\mathrm{j}} \in \mathfrak{R}_{\mathrm{n} \times \mathrm{n}}$ and $\Delta \mathrm{B}_{\mathrm{j}} \in \mathfrak{R}_{\mathrm{n} \times \mathrm{m}}$ are unknown but bounded structured uncertainty matrices corresponding to the effects of the uncertain parameters.

The uncertainty matrices can be rewritten in terms of the uncertain elements $\Delta_{\mathrm{al}}$ and $\Delta_{\mathrm{bl}}$ also the constant matrices $\mathrm{A}_{\mathrm{j}, \mathrm{i}} \in \mathfrak{R}_{\mathrm{n} \times \mathrm{n}}$ and $\mathrm{B}_{\mathrm{j}, \mathrm{i}} \in \mathfrak{R}_{\mathrm{n} \times \mathrm{m}}$ as:

$$
\begin{gathered}
\Delta \mathrm{A}_{\mathrm{j}}=\sum_{1-1}^{\mathrm{k}_{\mathrm{a}}} \Delta_{\mathrm{ai}} \mathrm{A}_{\mathrm{j}, \mathrm{l}}=\mathrm{M}_{\mathrm{j}, \mathrm{ac}}=\Delta_{\mathrm{j}, \mathrm{a}} \mathrm{N}_{\mathrm{j}, \mathrm{ar}} \\
\Delta \mathrm{B}_{\mathrm{j}}=\sum_{1-1}^{\mathrm{k}_{\mathrm{b}}} \Delta_{\mathrm{bi}} \mathrm{A}_{\mathrm{j}, \mathrm{l}}=\mathrm{M}_{\mathrm{j}, \mathrm{bc}}=\Delta_{\mathrm{j}, \mathrm{b}} \mathrm{N}_{\mathrm{j}, \mathrm{br}}
\end{gathered}
$$

From this representation and by letting $\mathrm{q}_{1} \stackrel{\Delta}{=} \operatorname{rank}\left(\mathrm{A}_{\mathrm{j}, 1}\right), \mathrm{p}_{1} \Delta \operatorname{\Delta rank}\left(\mathrm{B}_{\mathrm{j}, 1}\right)$ the constant matrices $\mathrm{M}_{\mathrm{j}, \mathrm{ac}}$, $\mathrm{M}_{\mathrm{j}, \mathrm{bc}}, \mathrm{N}_{\mathrm{j}, \text { ar }}$ and $\mathrm{N}^{\mathrm{j}, \mathrm{br}}$ are given by the equations:

$$
\begin{aligned}
& \mathrm{M}_{\mathrm{j}, \mathrm{ac}}=\left[\mathrm{M}_{\mathrm{ac}, 1}, \mathrm{M}_{\mathrm{ac}, 2}, \ldots, \mathrm{M}_{\mathrm{ac}, \mathrm{ka}}\right] \\
& \mathrm{M}_{\mathrm{j}, \mathrm{bc}}=\left[\mathrm{M}_{\mathrm{bc}, 1}, \mathrm{M}_{\mathrm{bc}, 2}, \ldots, \mathrm{M}_{\mathrm{bc}, \mathrm{kb}}\right] \\
& \mathrm{N}_{\mathrm{j}, \mathrm{ar}}=\left[\mathrm{N}_{\mathrm{ar}, 1}^{\mathrm{T}}, \mathrm{M}_{\mathrm{ar}, 2}^{\mathrm{T}}, \ldots, \mathrm{M}_{\mathrm{ar}, \mathrm{ka}}^{\mathrm{T}}\right] \\
& \mathrm{N}_{\mathrm{j}, \mathrm{br}}=\left[\mathrm{N}_{\mathrm{br}, 1}^{\mathrm{T}}, \mathrm{M}_{\mathrm{br}, 2}^{\mathrm{T}}, \ldots, \mathrm{M}_{\mathrm{br}, \mathrm{kb}}^{\mathrm{T}}\right]
\end{aligned}
$$

where, $\mathrm{M}_{\mathrm{ac}, 1} \in \mathfrak{R}^{\mathrm{n} \times \mathrm{q} 1}$ are the $\mathrm{q}_{1}$ nonzero column vectors of $\mathrm{A}_{\mathrm{j}, 1}, \mathrm{M}_{\mathrm{bc}, 1} \in \mathfrak{R}^{\mathrm{n} \times \mathrm{pl}}$ are the $\mathrm{p}_{1}$ nonzero column vectors of $\mathrm{B}_{\mathrm{j}, 1}$, while:

$$
\begin{aligned}
& \mathrm{N}_{\mathrm{ar}, 1}=\left(\mathrm{M}_{\mathrm{ac}, 1}^{\mathrm{T}} \mathrm{M}_{\mathrm{ac}, 1}\right)^{-1} \mathrm{M}_{\mathrm{ac}, 1}^{\mathrm{T}} \mathrm{A}_{\mathrm{j}, 1} \in \mathfrak{R}^{\mathrm{q} 1 \times \mathrm{n}}, \mathrm{N}_{\mathrm{br}, 1} \\
& =\left(\mathrm{M}_{\mathrm{bc}, 1}^{\mathrm{T}} \mathrm{M}_{\mathrm{bc}, 1}\right)^{-1} \mathrm{M}_{\mathrm{bc}, 1}^{\mathrm{T}} \mathrm{A}_{\mathrm{j}, 1} \in \mathfrak{R}^{\mathrm{pl \times m}}
\end{aligned}
$$


and $\Delta_{\mathrm{j}, \mathrm{a}}=$ block diag $\left[\Delta_{\mathrm{a} 1} \mathrm{I}_{\mathrm{q} 1}, \Delta_{\mathrm{a} 2} \mathrm{I}_{\mathrm{q} 2}, \ldots, \Delta_{\mathrm{a}_{\mathrm{ka}}} \mathrm{I}_{\mathrm{kas}}\right]$. With $\mathrm{q}^{1}$ and $p^{1}$, being $q^{1} \times q^{1}$ and $p^{1} \times p^{1}$ identity matrices respectively.

Without loss of generality, we can assume that $\left|\Delta_{\mathrm{ai}}\right| \leq 1$ and $\left|\Delta_{\mathrm{bj}}\right| \leq 1$ for $\mathrm{i}=1, \ldots, \mathrm{k}_{\mathrm{a}} ; \mathrm{j}=1, \ldots \ldots, \mathrm{k}_{\mathrm{b}}$.

So that one can rewrite the uncertain linear system in Eq. 10 as a nominal linear system with a disturbance input as shown below:

$$
\left(\begin{array}{l}
\dot{x}_{c}(t) \\
z_{c}(t) \\
y_{c}(t)
\end{array}\right)=\left(\begin{array}{ccc}
A_{o, j} & B_{i, j} & B_{o, j} \\
C_{1, j} & 0 & D_{1, j} \\
C_{o, j} & 0 & 0
\end{array}\right)\left(\begin{array}{c}
x_{c}(t) \\
\bar{w}_{c}(t) \\
u_{c}(t)
\end{array}\right)
$$

Where:

$$
\mathrm{B}_{\mathrm{l}, \mathrm{j}}=\left[\mathrm{M}_{\mathrm{j}, \mathrm{ac}}, \mathrm{M}_{\mathrm{j}, \mathrm{bc}}\right], \mathrm{C}_{1, \mathrm{j}}=\left[\mathrm{N}_{\mathrm{j}, \mathrm{ar}}^{\mathrm{T}} 0\right], \mathrm{D}_{\mathrm{l}, \mathrm{j}}=\left[\begin{array}{ll}
0 & \mathrm{~N}_{\mathrm{j}, \mathrm{br}}^{\mathrm{T}}
\end{array}\right] \text { and } \mathrm{C}_{\mathrm{o}, \mathrm{j}}=1 \text {. }
$$

From the uncertainty decomposition of (11-12) the fictitious disturbance input is given by $\overline{\mathrm{w}}_{\mathrm{c}}(\mathrm{t})=\operatorname{diag}\left[\Delta_{\mathrm{j}, \mathrm{a}}, \Delta_{\mathrm{j}, \mathrm{b}}\right] \mathrm{z}_{\mathrm{c}}(\mathrm{t})$ A controller for the disturbed system (13) can be constructed using the general feedback structure. Where the disturbed local linear model (13) corresponds to the system under consideration and the objective is to find the feedback controller, in the form:

$$
\left(\begin{array}{l}
\dot{x}_{c}(t) \\
u_{c}(t)
\end{array}\right)=\left(\begin{array}{ll}
A_{x, j} & C_{x, j} \\
C_{x, j} & D_{x, j}
\end{array}\right)\left(\begin{array}{l}
x_{c}(t) \\
y_{c}(t)
\end{array}\right)
$$

The closed-loop the system described in Eq. (1314) should be internally stable. Moreover, the effects of the disturbed input $\overline{\mathrm{w}}_{\mathrm{c}}(\mathrm{t})$ on the desired output $\mathrm{z}_{\mathrm{c}}(\mathrm{t})$, measured in terms of the infinity norm of their transfer function $\left\|\hat{\mathrm{T}} \mathrm{z}_{\mathrm{c}} \overline{\mathrm{w}}_{\mathrm{c}}(\mathrm{S})\right\| \infty$, should be less than a given bound $\gamma>0$. Consequently, the closed-loop system becomes:

$$
\begin{aligned}
& \left(\begin{array}{c}
\dot{\mathrm{x}}_{\mathrm{c}}(\mathrm{t}) \\
\mathrm{z}_{\mathrm{c}}(\mathrm{t})
\end{array}\right)=\left(\begin{array}{ll}
\mathrm{A}_{\mathrm{cl}} & \mathrm{B}_{\mathrm{cl}} \\
\mathrm{C}_{\mathrm{cl}} & \mathrm{D}_{\mathrm{cl}}
\end{array}\right)\left(\begin{array}{l}
\mathrm{x}_{\mathrm{c}}(\mathrm{t}) \\
\overline{\mathrm{w}}_{\mathrm{c}}(\mathrm{t})
\end{array}\right)
\end{aligned}
$$

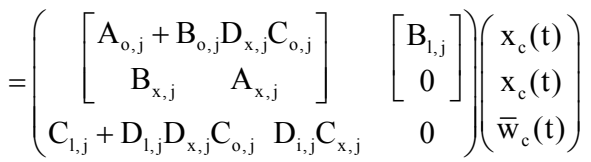

As proposed in reference (Ababneh et al., 2007), a static state feedback controller can be obtained by solving the following LMIs:

$$
\mathrm{Y}>0,\left(\begin{array}{ccc}
\left(\mathrm{A}_{\mathrm{o}, \mathrm{j}} \mathrm{Y}+\mathrm{B}_{\mathrm{o}, \mathrm{j}} \mathrm{M}\right)^{\mathrm{T}} & & \\
+\left(\mathrm{A}_{\mathrm{o}, \mathrm{j}} \mathrm{Y}+\mathrm{B}_{0, j} \mathrm{M}\right) & \mathrm{B}_{1, j} & \left(\mathrm{C}_{1, j} \mathrm{Y}+\mathrm{D}_{1, j} \mathrm{M}\right)^{\mathrm{T}} \\
\mathrm{B}_{1, j}^{\mathrm{T}} & -\gamma \mathrm{l} & 0 \\
\mathrm{C}_{1, j} \mathrm{Y}+\mathrm{D}_{1, j} \mathrm{M} & 0 & -\gamma \mathrm{l}
\end{array}\right)<0
$$

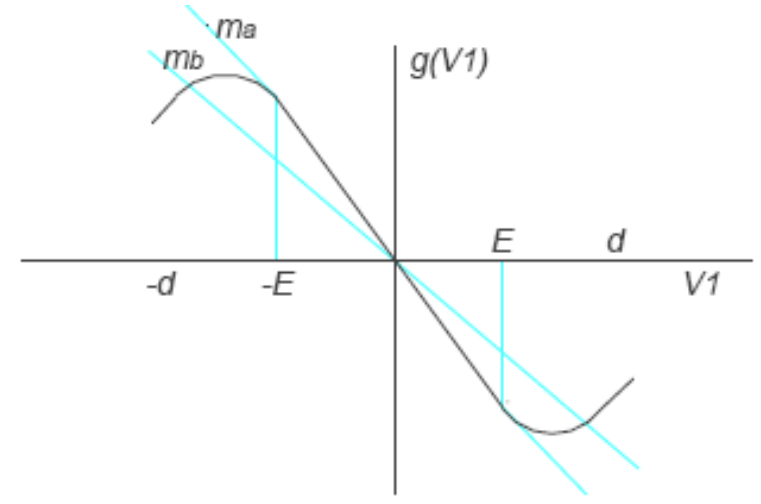

Fig. 4: Nonlinear representation of the Chua's resistor

A continuous-time state feedback controller for Eq. 1 can be constructed in the form:

$u_{c}(t)=-K_{c, j} x_{c}(t)$

where the feedback gain $\mathrm{K}_{\mathrm{c}, \mathrm{j}}$ is found from the solutions of (16) as $\mathrm{K}_{\mathrm{c}, \mathrm{j}}=-\mathrm{MY}^{-1}$ around each operating point.

Digital and observer based synchronization: In what follows, we present the observer based method and the digital redesign technique for the uncertain chaotic system at hand. Consider the following uncertain nonlinear system, which will be called the Drive-System:

$\dot{x}_{c}(t)=f\left(x_{c}(t), p\right), x_{c}(0)=x_{o}, y_{c}(t)=C_{c}(t)$

and a Response-System in the form:

$\dot{\hat{x}}_{\mathrm{c}}(\mathrm{t})=\hat{\mathrm{f}}\left(\hat{\mathrm{x}}_{\mathrm{c}}(\mathrm{t}), \mathrm{p}\right), \hat{\mathrm{x}}_{\mathrm{c}}(0)=\mathrm{x}_{\mathrm{o}}, \hat{\mathrm{y}}_{\mathrm{c}}(\mathrm{t})=\mathrm{C} \hat{\mathrm{x}}_{\mathrm{c}}(\mathrm{t})$

where, $f$ and $\mathrm{f}^{\mathrm{n}}$ are nonlinear functions, $\mathrm{x}_{\mathrm{c}}(\mathrm{t}) \in \mathfrak{R}^{\mathrm{n}}$ and $\hat{\mathrm{x}}_{\mathrm{c}}(\mathrm{t}) \in \mathfrak{R}^{\mathrm{n}}$ are the drive and response state variables, respectively. To achieve synchronization the $\lim _{x \rightarrow \infty}\left\|x_{c}(t)-\hat{x}_{c}(t)\right\|=0$ must be satisfied. Furthermore, the drive system can be expressed as:

$\dot{\mathrm{x}}_{\mathrm{c}}(\mathrm{t})=\tilde{\mathrm{A}}_{\mathrm{j}} \mathrm{x}_{\mathrm{c}}(\mathrm{t})=\left(\mathrm{A}_{\mathrm{o}, \mathrm{j}} \pm \Delta \mathrm{A}_{\mathrm{j}}\right) \mathrm{x}_{\mathrm{c}}(\mathrm{t})$

Using the uncertainty decomposition techniques, the uncertain local linear model in (23) can be rewritten as:

$$
\left(\begin{array}{l}
\dot{x}_{c}(t) \\
z_{c}(t) \\
y_{c}(t)
\end{array}\right)=\left(\begin{array}{cc}
A_{o, j} & B_{1, j} \\
C_{1, j} & 0 \\
C & 0
\end{array}\right)\left(\begin{array}{l}
x_{c}(t) \\
\bar{w}_{c}(t)
\end{array}\right)
$$


where, $B_{1, j}=\left[M_{j, a c}\right], C_{1, j}=[N]$ and $\bar{w}_{c}(t)=\Delta_{j, a} z_{c}(t)$.

An $H \infty$ robust observer for (21) can be designed using the output-injection structure:

$\dot{\hat{x}}_{\mathrm{c}}(\mathrm{t})=\mathrm{A}_{\mathrm{o}, \mathrm{j}} \hat{\mathrm{x}}(\mathrm{t})+\mathrm{K}_{\mathrm{j}, \mathrm{a}}\left(\mathrm{y}_{\mathrm{c}}(\mathrm{t})-\hat{\mathrm{y}}_{\mathrm{c}}(\mathrm{t})\right)$

with observer error defined as:

$\dot{\mathrm{e}}_{\mathrm{c}}(\mathrm{t})=\dot{\mathrm{x}}_{\mathrm{c}}(\mathrm{t})-\dot{\hat{\mathrm{x}}}_{\mathrm{c}}(\mathrm{t})$

The error dynamics are found to be:

$\dot{\mathrm{e}}_{\mathrm{c}}(\mathrm{t})=\left(\mathrm{A}_{\mathrm{o}, \mathrm{j}}-\mathrm{K}_{\mathrm{j}, \mathrm{C}} \mathrm{C}\right) \mathrm{e}_{\mathrm{c}}(\mathrm{t})+\mathrm{B}_{\mathrm{i}, \mathrm{j}} \overline{\mathrm{w}}_{\mathrm{c}}(\mathrm{t})$

The error dynamics will then have an $\mathrm{H} \infty$ robust performance given by $\mathrm{K}_{\mathrm{j}, \mathrm{o}}=\mathrm{N}_{\mathrm{j}, \mathrm{0}} \mathrm{X}^{-1}$ where $\mathrm{X}=\mathrm{X}^{\mathrm{T}}>0$ and $\mathrm{N}_{\mathrm{j}, \mathrm{o}}$ are the matrix solutions of the following LMI problem:

$$
\left(\begin{array}{ccc}
A_{o, j}^{T} X+X A_{o, j}-C^{T} N_{j, o}^{T}-N_{j, o} C & X_{1, j} & 1_{n} \\
B_{i, j}^{T} X & -\sigma l & 0 \\
1_{n} & 0 & -\sigma l
\end{array}\right)<0
$$

Consider that the objective is to design a digital observer in the form:

$$
\begin{aligned}
& \hat{\mathrm{x}}_{\mathrm{d}}(\mathrm{kT})=\mathrm{G}_{\mathrm{o}} \hat{\mathrm{x}}_{\mathrm{d}}(\mathrm{kT}-\mathrm{T})+\mathrm{H}_{\mathrm{o}} \mathrm{u}_{\mathrm{c}}(\mathrm{kT}-\mathrm{T}) \\
& +\mathrm{L}_{\mathrm{j}, \mathrm{d}}\left[\mathrm{y}_{\mathrm{c}}(\mathrm{kT}-\mathrm{T})+\mathrm{y}_{\mathrm{c}}(\mathrm{kT})\right]
\end{aligned}
$$

Such that the digital states of (26) closely match the states of the continuous-time observer. For the nominal part of system (18), the continuous-time error dynamics become:

$\dot{\mathrm{e}}_{\mathrm{c}}(\mathrm{t})=\mathrm{A}\left(\mathrm{A}_{\mathrm{o}, \mathrm{j}}-\mathrm{K}_{\mathrm{j}, \mathrm{o}} \mathrm{C}\right) \mathrm{e}_{\mathrm{c}}(\mathrm{t})$

where the digital error is defined as $e_{d}(k T)=x_{d}(k T)-\hat{x}_{d}(k T)$. The error dynamics in can be seen as a feedback-controlled system of the form:

$\dot{\mathrm{e}}_{\mathrm{c}}(\mathrm{t})=\mathrm{A}_{\mathrm{o}, \mathrm{j}} \mathrm{e}_{\mathrm{c}}(\mathrm{t})+\mathrm{l}_{\mathrm{n}} \mathrm{u}_{\mathrm{e}}(\mathrm{t})$

with $u_{e}(t)=-K_{j, 0} C e_{c}(t)$.

And this can be represented as:

$\mathrm{e}_{\mathrm{d}}(\mathrm{kT}-\mathrm{T})=(\mathrm{G}-\mathrm{MN}) \mathrm{e}(\mathrm{kT})$ where, $\quad \mathrm{G}=\mathrm{e}^{\mathrm{A}_{\mathrm{o}, \mathrm{T}} \mathrm{T}}, \mathrm{M}=\left(\mathrm{G}-\mathrm{1}_{\mathrm{n}}\right) \mathrm{A}_{\mathrm{o}, \mathrm{j}}^{-1} \mathrm{~K}_{\mathrm{j}, \mathrm{o}} \quad$ and $\mathrm{N}=\left(\mathrm{l}_{\mathrm{d}}+\mathrm{CM}\right)^{-1} \mathrm{CG}$.

Let $\mathrm{L}_{\mathrm{j}, \mathrm{d}}=\mathrm{M}\left(1_{\mathrm{m}}+\mathrm{CM}\right)^{-1}$ one gets $\mathrm{MN}=\mathrm{L}_{\mathrm{j}, \mathrm{d}} \mathrm{CG}$ and the error dynamics become:

$\mathrm{x}_{\mathrm{d}}(\mathrm{kT}+\mathrm{T})-\hat{\mathrm{x}}_{\mathrm{d}}(\mathrm{kT}+\mathrm{T})+$

$\left(\mathrm{G}-\mathrm{L}_{\mathrm{j}, \mathrm{d}} \mathrm{CG}\right)\left[\mathrm{x}_{\mathrm{d}}(\mathrm{kT})-\hat{\mathrm{x}}_{\mathrm{d}}(\mathrm{kT})\right]$

Then substituting the following equations:

$\mathrm{x}_{\mathrm{d}}(\mathrm{kT}+\mathrm{T})=\mathrm{Gx}_{\mathrm{d}}(\mathrm{kT})+\mathrm{Hu}_{\mathrm{d}}(\mathrm{kT})$,

$\mathrm{y}_{\mathrm{c}}(\mathrm{kT})=\mathrm{y}_{\mathrm{d}}(\mathrm{kT})=\mathrm{Cx}_{\mathrm{d}}(\mathrm{kT})$

And:

$\mathrm{CGx}_{\mathrm{d}}(\mathrm{kT})=\mathrm{Cx}_{\mathrm{d}}(\mathrm{kT}+\mathrm{T})-\mathrm{CHu}_{\mathrm{d}}(\mathrm{kT})$

$=y_{d}(k T+T)-C_{d} u_{d}(k T)$

This produces the final observer equation:

$\hat{\mathrm{x}}_{\mathrm{d}}(\mathrm{kT})=\mathrm{G}_{0} \hat{\mathrm{x}}_{\mathrm{d}}(\mathrm{kT}-\mathrm{T})+\mathrm{Hu}_{\mathrm{d}}(\mathrm{kT}-\mathrm{T})$
$+\mathrm{L}_{\mathrm{j}, \mathrm{d}}\left[\mathrm{y}_{\mathrm{c}}(\mathrm{kT}-\mathrm{T})+\mathrm{y}_{\mathrm{c}}(\mathrm{kT})\right]$

where, $\mathrm{G}_{\mathrm{o}}=\mathrm{G}-\mathrm{L}_{\mathrm{j}, \mathrm{d}} \mathrm{CG}$

$$
\begin{aligned}
\hat{\mathrm{x}}_{\mathrm{d}}(\mathrm{kT}) & =\mathrm{G}_{\mathrm{o}} \hat{\mathrm{x}}_{\mathrm{d}}(\mathrm{kT}-\mathrm{T})+\mathrm{H}_{\mathrm{o}} \mathrm{u}_{\mathrm{c}}(\mathrm{kT}-\mathrm{T}) \\
& +\mathrm{L}_{\mathrm{j}, \mathrm{d}}\left[\mathrm{y}_{\mathrm{c}}(\mathrm{kT}-\mathrm{T})+\mathrm{y}_{\mathrm{c}}(\mathrm{kT})\right] \\
\text { and } \mathrm{H}_{\mathrm{o}} & =\mathrm{H}-\mathrm{L}_{\mathrm{j}, \mathrm{d}} \mathrm{CH} .
\end{aligned}
$$

Numerical simulation: Here, the proposed methodology will be used to perform digital synchronization for two benchmark Chua's chaotic systems. Given the following Chua system where parameters $\alpha$ and $\beta$ are assumed to be uncertain but bound to a given interval:

$$
\left[\begin{array}{c}
\dot{\mathrm{x}}_{\mathrm{c} 1}(\mathrm{t}) \\
\dot{\mathrm{x}}_{\mathrm{c} 2}(\mathrm{t}) \\
\dot{\mathrm{x}}_{\mathrm{c} 3}(\mathrm{t})
\end{array}\right]=\left[\begin{array}{c}
\tilde{\mathrm{a}}\left[\mathrm{x}_{\mathrm{c} 2}(\mathrm{t})-\mathrm{x}_{\mathrm{c} 1}(\mathrm{t})-\mathrm{g}_{\mathrm{NL}}\left(\mathrm{x}_{\mathrm{c} 1}(\mathrm{t})\right)\right] \\
\mathrm{x}_{\mathrm{cl}}(\mathrm{t})-\mathrm{x}_{\mathrm{c} 2}(\mathrm{t})+\mathrm{x}_{\mathrm{c} 3}(\mathrm{t}) \\
-\tilde{\mathrm{b}} \mathrm{x}_{\mathrm{c} 2}(\mathrm{t})
\end{array}\right]+\tilde{\mathrm{B}}\left[\begin{array}{c}
\mathrm{u}_{\mathrm{c}, 1}(\mathrm{t}) \\
\mathrm{u}_{\mathrm{c}, 2}(\mathrm{t}) \\
\mathrm{u}_{\mathrm{c}, 3}(\mathrm{t})
\end{array}\right]
$$

The linear model of Eq. 32 can be rewritten using the fuzzy modeling as:

$$
\dot{x}_{c}(t)=\left\{\begin{array}{c}
A_{j}^{* 1}[\tilde{a}, \tilde{b}] x_{c}(t)+B_{j}^{*}\left[\tilde{B}_{j}\right] u_{c}(t),(\text { near } 0) \\
A_{j}^{* 2}[\tilde{a}, \tilde{b}] x_{c}(t)+B_{j}^{*}\left[\tilde{B}_{j}\right] u_{c}(t),(\text { near } \pm d)
\end{array}\right.
$$

Here $\mathrm{j} \tilde{\mathrm{B}}_{\mathrm{j}}$ refers to the uncertainty of the input matrix of the local linear system, which will be treated as an 202 
extra uncertain parameter. Rewriting the uncertain parameters $\left(\tilde{a}, \tilde{\beta}, \tilde{B}_{j}\right) \quad$ as $\quad \tilde{a}=a_{0}+\Delta a_{1}, \tilde{\beta}=\beta_{0}+\Delta \beta_{1} \quad$ and $\tilde{\mathrm{B}}_{\mathrm{j}}=\mathrm{B}_{0}+\Delta \mathrm{B}_{1}$ where $\mathrm{a}_{0}=\frac{19}{2}, \beta_{0}=\frac{100}{7}, 1_{3 \times 3}, \mathrm{a}_{1}=\frac{19}{40}, \beta_{1}=\frac{5}{7}$ and $B_{1}=\frac{5}{100}$. Then the linear model of the uncertain Chua's circuit can be written as:

$$
\dot{x}_{c}(t)=\left\{\begin{array}{l}
\left(A_{o, j}^{* 1}+\Delta A_{1, j}^{* 1}\right) x_{c}(t)+\left(B_{o, j}^{*}+\Delta B_{1, j}^{* 1}\right) \\
u_{c}(t),(\text { near } 0) \\
\left(A_{o, j}^{* 2}+\Delta A_{1, j}^{* 2}\right) x_{c}(t)+\left(B_{o, j}^{*}+\Delta B_{1, j}^{* 1}\right) \\
u_{c}(t),(\text { near } \pm d)
\end{array}\right.
$$

with $A_{o, j}^{* 1}=A_{j}^{* 1}\left[a_{0}, b_{0}\right], A_{o, j}^{* 2}=A_{j}^{* 2}\left[a_{0}, b_{0}\right]$ and $B_{o, j}^{*}=B_{j}^{*}\left[B_{0}\right]$. Note that both piecewise and nonlinear Chua's circuits has the same treatment since they differ only in the item $1_{1 \mathrm{i}}$ of the system matrix A. Using the decomposition method presented earlier, the uncertainties of equation (34) can be decomposed into the following matrices:

$$
\begin{aligned}
& M_{\mathrm{ac}, 1}^{*}=\mathrm{M}_{\mathrm{ac}, 2}^{*}=\mathrm{M}_{\mathrm{ac}, 3}^{*}=\left\{\begin{array}{l}
\left\{\left[\begin{array}{c}
-\mathrm{a}_{1}\left(\mathrm{~g}_{\mathrm{b}}-\mathrm{g}_{\mathrm{a}}\right) \\
0 \\
0
\end{array}\right]\right\} \text { for piecewise } \\
\left\{\left[\begin{array}{c}
-\mathrm{a}_{1}\left(\mathrm{~g}_{2}-\mathrm{g}_{1}\right) \\
0 \\
0
\end{array}\right]\right\} \text { for nonlinear }
\end{array}\right\} \\
& \mathrm{N}_{\mathrm{ar}, 1}^{*}=\mathrm{N}_{\mathrm{ar}, 2}^{*}=\mathrm{N}_{\mathrm{ar}, 3}^{*}=\left[\begin{array}{lll}
1 & 0 & 0 \\
0 & 1 & 0
\end{array}\right] \text {, } \\
& \mathrm{N}_{\mathrm{br}, 1}^{*}=\mathrm{N}_{\mathrm{br}, 2}^{*}=\mathrm{N}_{\mathrm{br}, 3}^{*}=1 \\
& \mathrm{M}_{\mathrm{ac}, 1}^{*}=\mathrm{M}_{\mathrm{ac}, 2}^{*}=\mathrm{M}_{\mathrm{ac}, 3}^{*}=
\end{aligned}
$$

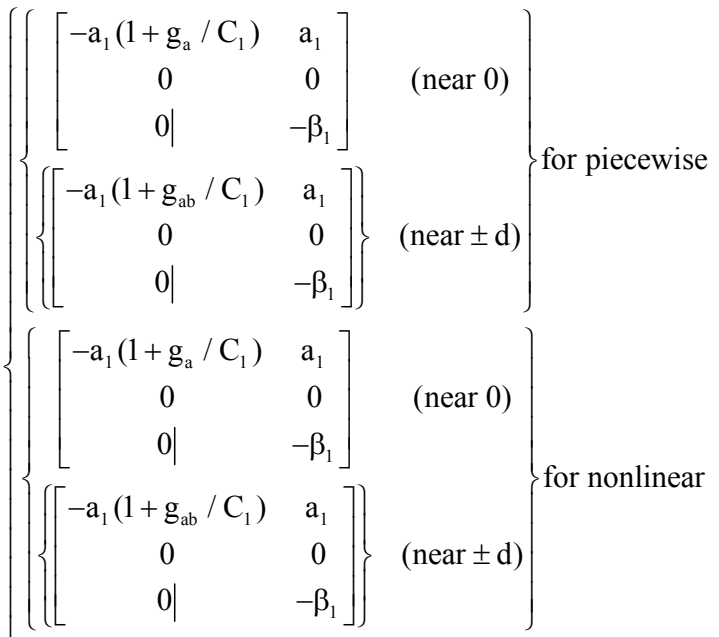

Methodology: The synchronization method can be summarized as follows:

Firstly: the fuzzy modeling of chaotic systems using the Takagi-Sugeno (TS) model is derived.

Secondly: the uncertainty decomposition is worked out by incorporating the uncertainties of the chaotic system in the fuzzy linear model. Where the uncertain chaotic system is expressed as set of linear models.

Thirdly: the digital and observer-based synchronization is performed for each linear model synchronization performed as the solution to a linear matrix inequality problem.

Note that the synchronization phenomenon may be viewed as an observer design problem; so that the error signal converges to zero globally and asymptotically, in this way the response system is synchronized to the drive system.

Results and discussions: The robust synchronization for the chaotic Chua's circuit with a piecewise-linear negative resistance is presented in Fig. 5. The output matrix is expressed as $\mathrm{C}=[1,0,0]$. The simulations were carried out with SIMULINK of MATLAB using a fifth-order Dormand-Prince algorithm with a fixed integration step of $\tau=0.001$ and performance bound of $\sigma=0.1$ for the continuous-time simulation and a sampled-hold period of $\mathrm{T}=0.1$ for the discrete-time simulation. The simulation results shown in the Fig. 5.
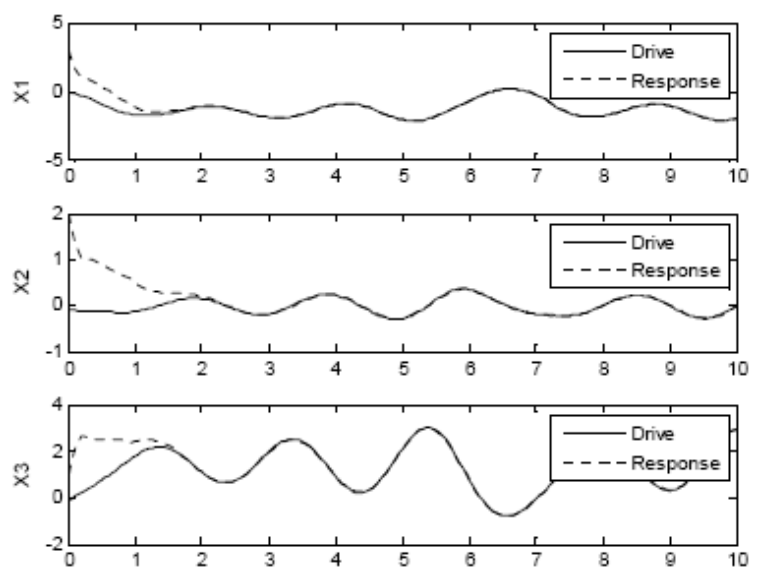

Fig. 5: Performance of synchronization for the piecewise-linear Chua's circuit 

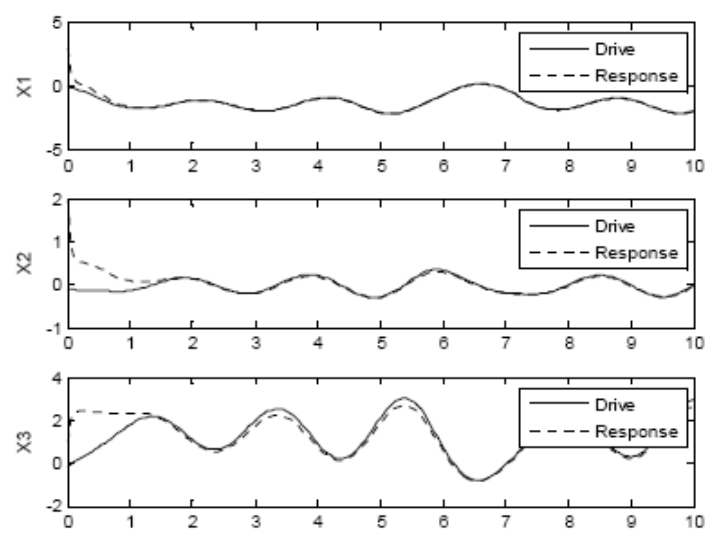

Fig. 6: Performance of synchronization for the nonlinear Chua's circuit

Furthermore, using the same MATLAB parameters and sample and a sampled-hold period, the robust synchronization for the chaotic Chua's circuit with a nonlinear negative resistance is presented in Fig. 6.

Figures 5 and 6 demonstrated the effectiveness of the methodology presented in this study. The response (receiver) system was able to synchronize very closely with drive (transmitter) system. Furthermore, both piecewise linear and nonlinear uncertain Chua circuits synchronized wonderfully in the three trajectories with insignificant errors.

\section{CONCLUSION}

This chaos synchronization method consists of designing a digital system (receiver) to synchronize with a given continuous-time chaotic system (transmitter). The chaos synchronization was derived based on the classical observer approach to synchronization, where the synchronization is achieved by making the error dynamics asymptotically stable about zero. As shown by convincing numerical results on uncertain chaotic systems, this new methodology is capable of achieving excellent synchronization performances, even in the presence of significant parametric uncertainties for uncertain chaos systems. The Takagi-Sugeno (TS) fuzzy model was used to model the chaotic dynamic system, while the uncertainties are decomposed such that the uncertain chaotic system was rewritten as a set of local linear models with an additional disturbed input.

\section{REFERENCES}

Ababneh, M., A. Almanasreh and H. Amasha, 2009, Design of digital controllers for uncertain chaotic systems using fuzzy logic. J. Franklin Inst., 346: 543-556. DOI: 10.1016/j.jfranklin.2009.02.002
Ababneh, M., J. Ramírez, G. Chen and L. Shieh, 2007. Robust digital controllers for uncertain chaotic systems: A digital redesign approach. Chaos, Solitons Fractals, 31: 1149-1164. DOI: 10.1016/j.chaos.2005.08.217

Ahmad, M.A. and Z. Mohamed, 2009. Hybrid fuzzy logic control with input shaping for input tracking. Am. J. Eng. Applied Sci., 2: 241-251. ISSN: 1941-7020

Bernal, M., T.M. Guerra and A. Kruszewski, 2009. A membership-function-dependent approach for stability analysis and controller synthesis of TakagiSugeno models. Fuzzy Sets Syst., 160: 2776-2795. DOI: 10.1016/j.fss.2009.02.005

Blekhman, I.I., A.L. Fradkov, O.P. Tomchina and D.E. Bogdanov, 2002. Self-synchronization and controlled synchronization: General definition and example design. Math. Comput. Simulation, 58: $\quad 367-384$. DOI: $10.1016 / \mathrm{S} 0378-$ 4754(01)00378-0

Bowong, S., M. Kakmeni and R. Koina, 2006. Chaos synchronization and duration time of a class of uncertain chaotic systems. Math. Comput. Simulation, $\quad 71$ : 212-228. DOI: 10.1016/j.matcom.2006.01.006

Lu, J., F. Han, X. Yu, G. Chen, 2004. Generating 3-D multi-scroll chaotic attractors: A hysteresis series switching method. Automatica, 40: 1677-1687. DOI: 10.1016/j.automatica.2004.06.001

Fujioka, H., C.Y. Kao, S. Almér and U. Jönsson, 2009. Robust tracking with $\mathrm{H}_{\infty}$ performance for PWM systems. Automatica, 45: 1808-1818. DOI: 10.1016/j.automatica.2009.03.026

Gámez-Guzmán, L. C. Cruz-Hernández, R.M. LópezGutiérrez and E.E. García-Guerrero, 2009. Synchronization of Chua's circuits with multiscroll attractors: Application to communication. Commun. Nonlinear Sci. Numerical Simulation, 14: 2765-2775. DOI: 10.1016/j.cnsns.2008.10.009

Hafaifa, A., F. Laaouad and M. Guemana, 2009. A new engineering method for fuzzy reliability analysis of surge control in centrifugal compressor. Am. J. Eng. Applied Sci., 2: 676-682. DOI: 10.3844/ajeassp.2009.676.682

Hassan, H., M. Moghavvemi and A. Mohamed, 2010. Takagi-sugeno fuzzy gains scheduled pi controller for enhancement of power system stability. Am. J. Applied Sci., 7: 145-152. DOI: 10.3844/ajassp.2010.145.152

Nijmeijer, H., 2001. A dynamical control view on synchronization. Physica D: Nonlinear Phenomena, 154: 219-228. DOI: $10.1016 / \mathrm{S} 0167-$ 2789(01)00251-2 
Nijmeijer, H., I.I. Blekhman, A.L. Fradkov and P.A. Yu, 1997. Self-synchronization and controlled synchronization. Proceedings of 1st International Conference on Control of Oscillations and Chaos, Aug. 27-29, St. Petersburg, Russia, pp: 36-41. DOI: $10.1109 / C O C .1997 .633464$

Shieh, L.S., W.M. Wang and M.K.A. Panicker, 1998. Design of PAM and PWM digital controllers for cascaded analog systems. ISA Trans., 37: 201-213. DOI: 10.1016/S0019-0578(98)00026-3

Sung, H.C., D.W. Kim, J.B. Park and Y.H. Joo, 2010. Robust digital control of fuzzy systems with parametric uncertainties: LMI-based digital redesign approach. Fuzzy Sets Syst., 161: 919-933. DOI: 10.1016/j.fss.2009.04.016
Xie, Q., G. Chen and E.M. Bollt, 2002. Hybrid chaos synchronization and its application in information processing. Math. Comput. Modell., 35: 145-163. DOI: $10.1016 / \mathrm{S} 0895-7177(01) 00157-1$

Yau, H.T. and J.J. Yan, 2008. Chaos synchronization of different chaotic systems subjected to input nonlinearity. Applied Math. Comput., 197: 775-788. DOI: 10.1016/j.amc.2007.08.014 